\title{
O 034 - Variation of the sagittal vertical axis during walking and its determinants
}

\author{
A. Assi ${ }^{\mathrm{a}, \mathrm{b}, *}$, Z. Bakouny ${ }^{\mathrm{a}}$, A.J. Bizdikian ${ }^{\mathrm{a}}$, J. Otayek ${ }^{\mathrm{a}}, \mathrm{F}$. Yared $^{\mathrm{a}}$, V. Lafage ${ }^{\mathrm{c}}$, N. Khalil $^{\mathrm{a}}$, \\ A. Massaad ${ }^{\text {a }}$, I. Ghanem ${ }^{\text {a }}$, W. Skalli ${ }^{\text {b }}$

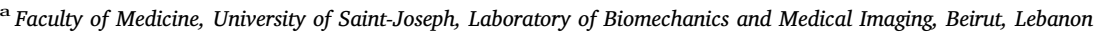 \\ ${ }^{\mathrm{b}}$ Arts et Métiers ParisTech, Institut de Biomécanique Humaine Georges Charpak, Paris, France \\ ${ }^{\mathrm{c}}$ Hospital for Special Surgery, Spine Division, New York, USA
}

Keywords:

Sagittal vertical axis

Gait analysis

Biplanar X-rays

Image registration

\section{Introduction}

Patients with adult spinal deformities (ASD) are known to have altered postural alignment affecting their quality of life and activities of daily living, especially gait. The Sagittal Vertical Axis (SVA), a postural parameter calculated as the distance between the posterior corner of the sacrum and the C7-plumbline on full-body sagittal radiographs [1], has been shown to be highly altered in ASD. Even though this parameter is positional and could vary during gait, no studies have investigated its variation during walking even in asymptomatic subjects.

\section{Research question}

Is SVA modified during gait in asymptomatic subjects?

\section{Methods}

75 asymptomatic adults (age: $28 \pm 11$ years [18-59],32 F) underwent gait analysis with reflective markers on the pelvis, lower limbs(LL) [2] and the C7 spinous process. Kinematics of the pelvis and LL were extracted. Subjects then underwent low-dose full-body biplanar radiographs in standing position, with the reflective markers still in place. Subject-specific 3D spino-pelvic, acetabular and LL parameters were calculated as well as the 3D radiological SVA (rSVA). Then, the 3D bones were registered on each frame of the gait cycle [3] (Fig.1). A new technique developed for the purpose of this study, utilizing finite element modeling, was used to reduce soft tissue artefacts. The SVA was then computed during the gait cycle, using the 3D registered bones, at each time frame: average (av_SVA), range of motion (ROM_SVA) and the difference between dynamic and static SVA $(\triangle S V A=$ av_SVA- rSVA) were calculated. In order to investigate the determinants of static and dynamic SVA parameters, a univariate analysis (Pearson's correlations) followed by a multivariate analysis (stepwise-multiple-linear regressions) were performed; the dependent variables were demographics, 3D kinematics and 3D skeletal parameters (spino-pelvic, acetabular and LL).

\section{Results}

The rSVA ranged from -66 to $38 \mathrm{~mm}$ with a mean of $-9 \mathrm{~mm}$. The av_SVA (ROM_SVA) during gait ranged from 9 to $108 \mathrm{~mm}$ ( 9 to $45 \mathrm{~mm}$ ) with a mean of $46 \mathrm{~mm}$ ( $23 \mathrm{~mm}$, respectively). $\triangle$ SVA ranged from 16 to $120 \mathrm{~mm}$ with a mean of $55 \mathrm{~mm}$. Fig. 2 shows the SVA variation during gait relative to its radiological value. The multivariate analysis showed (Fig.3) that: rSVA (R2 $=0.2$ ) was solely determined by sex (F:- $20 \mathrm{~mm}$ vs $\mathrm{M}: 1 \mathrm{~mm}, \mathrm{p}<0.001)$; av_SVA $(\mathrm{R} 2=0.4)$ during gait was determined by weight $(\beta=0.97, \mathrm{p}<0.001)$ and rSVA $(\beta=0.18, \mathrm{p}<0.001)$; ROM_SVA $(R 2=0.2)$ was determined by acetabular abduction $(\beta=$ $0.52, \mathrm{p}=0.038)$ and ROM hip flexion/extension during gait $(\beta=1.48$, $\mathrm{p}<0.001) ; \quad \Delta$ SVA was solely determined by lumbar lordosis $(\mathrm{r}=0.33, \mathrm{p}=0.004)$.

\footnotetext{
* Corresponding author at: Faculty of Medicine, University of Saint-Joseph, Laboratory of Biomechanics and Medical Imaging, Beirut, Lebanon.

E-mail address: ayman.assi@gmail.com (A. Assi).
} 


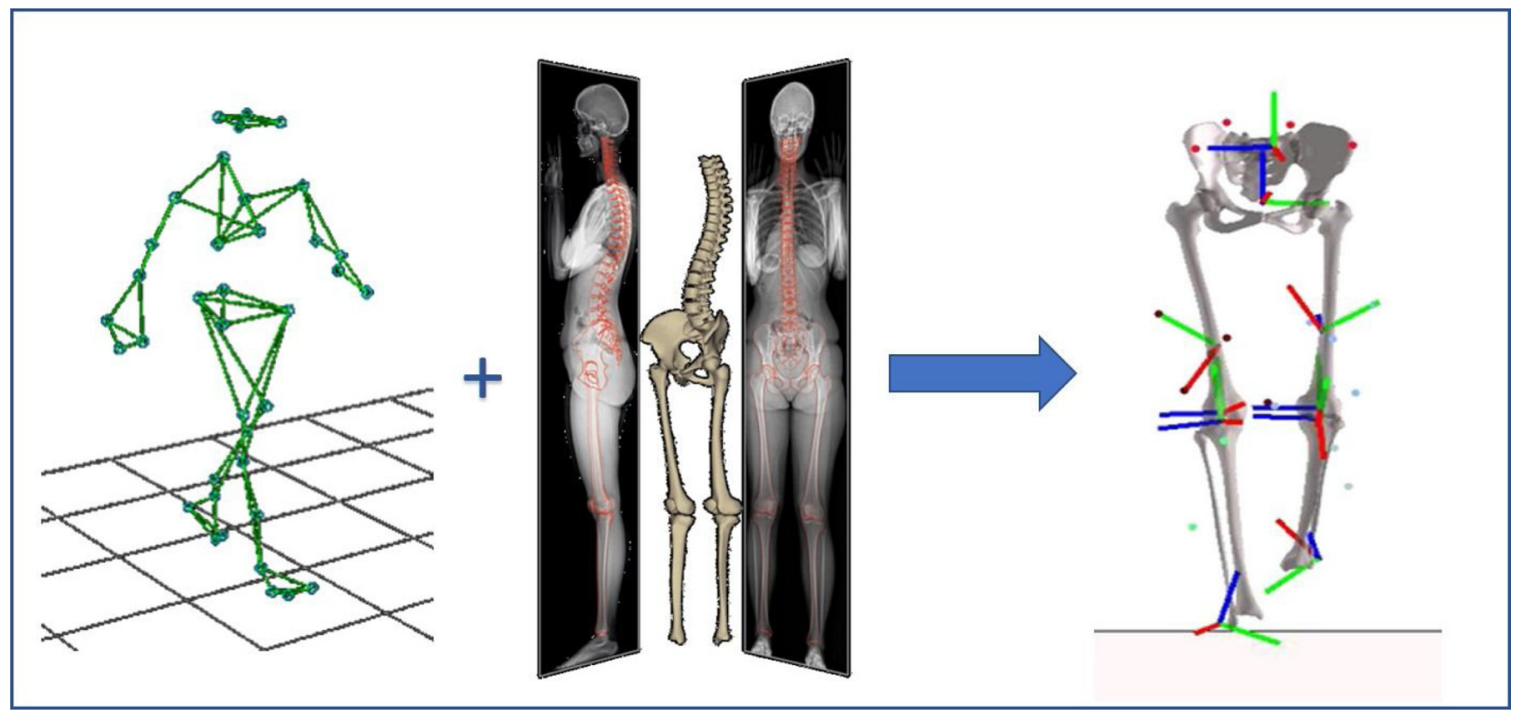

Fig. 1. Registration of 3D skeletal reconstructions from biplanar X-rays with 3D gait analysis.

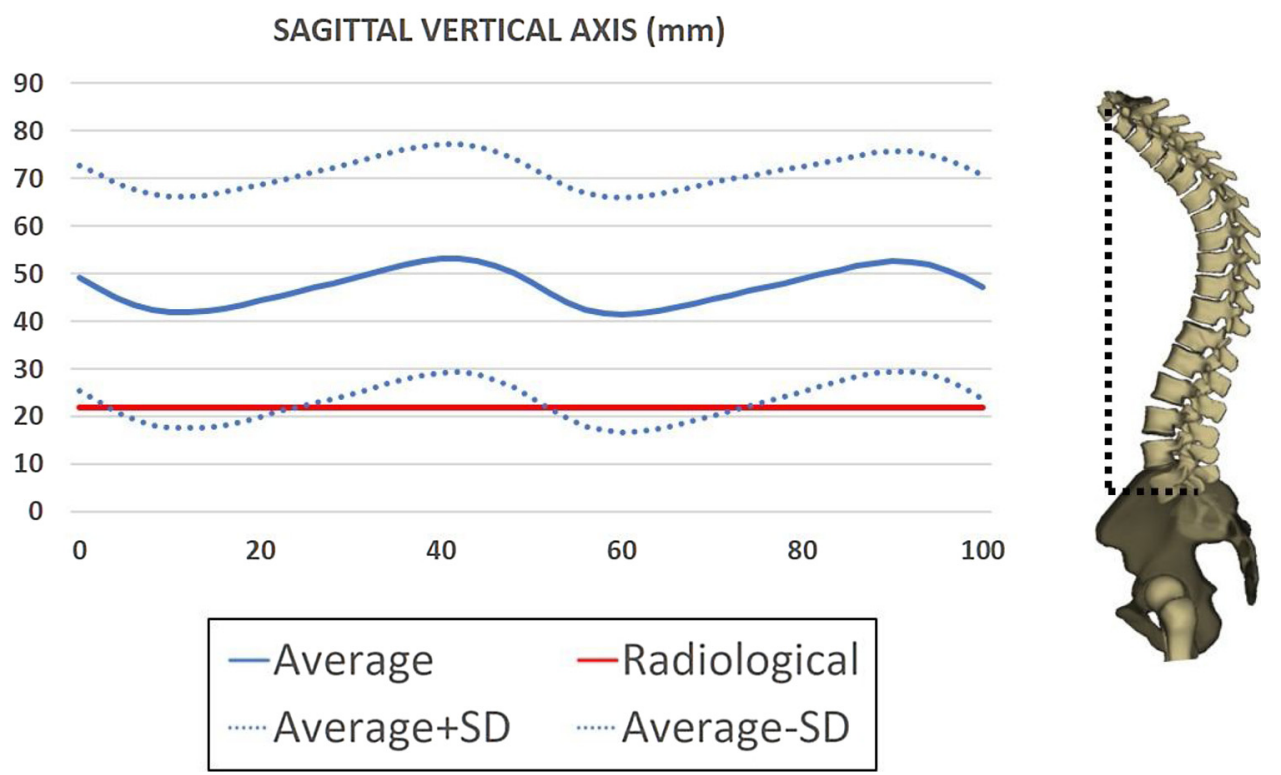

Fig. 2. Variation of the Sagittal Vertical Axis during walking in asymptomatic subjects in regards to its radiological value. 


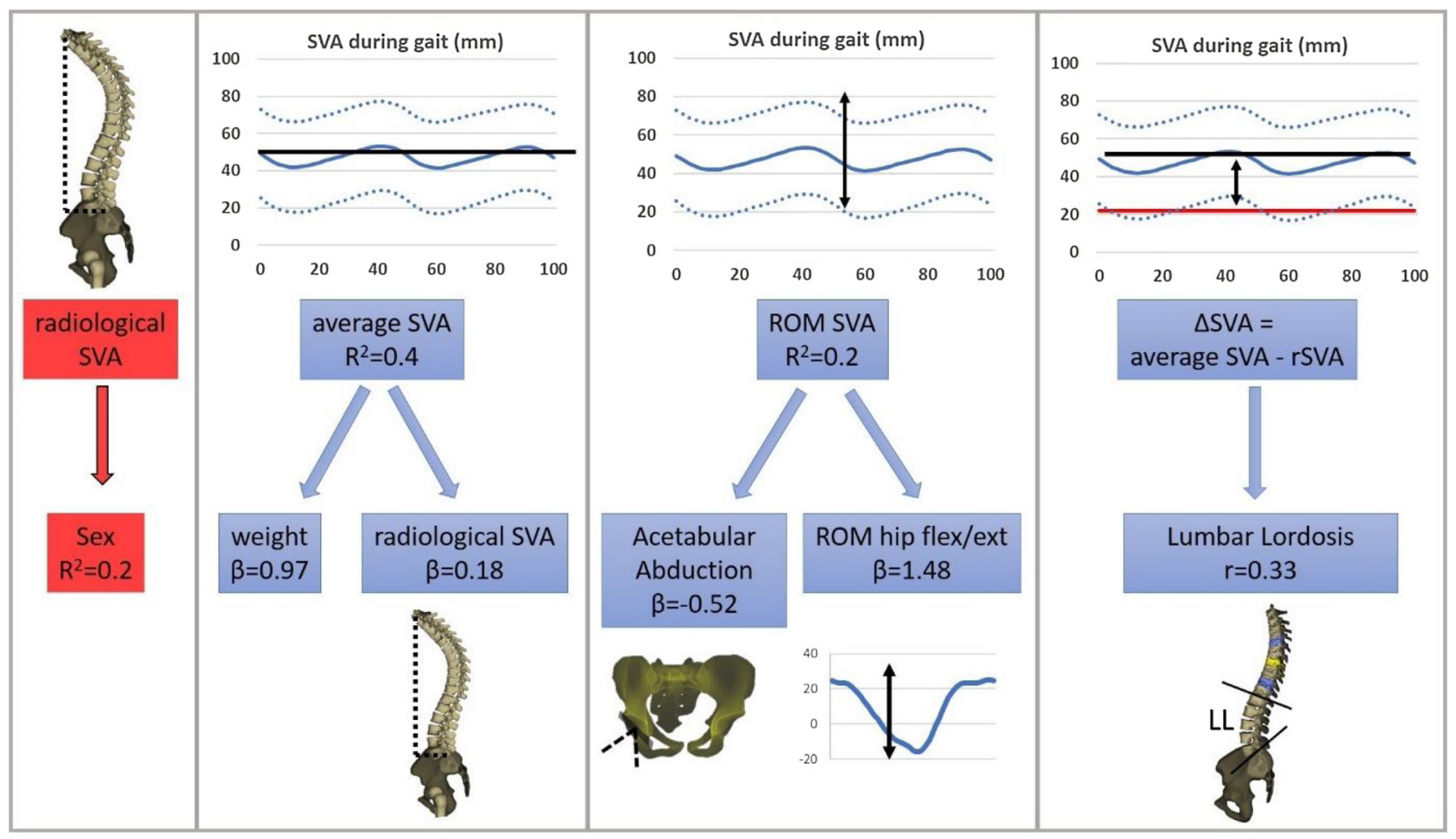

Fig. 3. Demographic, skeletal and kinematic determinants of radiological and dynamic Sagittal Vertical Axis.

\section{Discussion}

This is the first study to evaluate SVA during walking in asymptomatic subjects. In general, subjects tended to bend their trunk forward during gait (positive $\triangle$ SVA), especially those with higher lumbar lordosis and higher rSVA. Patients with ASD, who are known to have an increased rSVA, might show a more forwarded trunk during gait which could possibly increase their risk of falling.

\section{References}

[1] Jackson (1994).

[2] Davis (1990).

[3] Söderkvist (1993). 